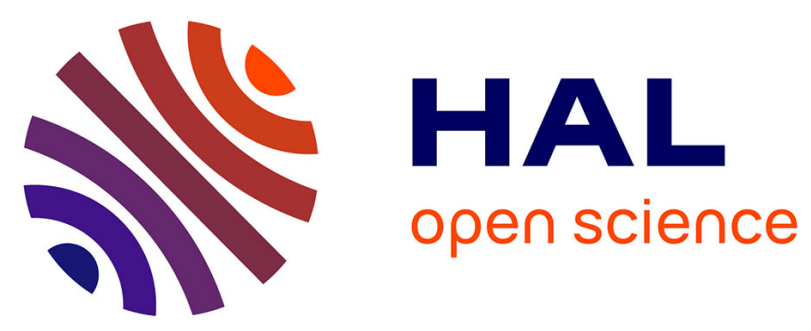

\title{
Active lighting applied to three-dimensional reconstruction of specular metallic surfaces by polarization imaging
}

Olivier Morel, Christophe Stolz, Fabrice Mériaudeau, Patrick Gorria

\section{- To cite this version:}

Olivier Morel, Christophe Stolz, Fabrice Mériaudeau, Patrick Gorria. Active lighting applied to threedimensional reconstruction of specular metallic surfaces by polarization imaging. Journal of Optics A: Pure and Applied Optics, 2006, 45 (17), pp.4062-4068. hal-00514527

\author{
HAL Id: hal-00514527 \\ https://hal.science/hal-00514527
}

Submitted on 2 Sep 2010

HAL is a multi-disciplinary open access archive for the deposit and dissemination of scientific research documents, whether they are published or not. The documents may come from teaching and research institutions in France or abroad, or from public or private research centers.
L'archive ouverte pluridisciplinaire HAL, est destinée au dépôt et à la diffusion de documents scientifiques de niveau recherche, publiés ou non, émanant des établissements d'enseignement et de recherche français ou étrangers, des laboratoires publics ou privés. 


\title{
Active lighting applied to three-dimensional reconstruction of specular metallic surfaces by polarization imaging
}

\author{
Olivier Morel, Christophe Stolz, Fabrice Meriaudeau, and Patrick Gorria
}

\begin{abstract}
In the field of industrial vision, the three-dimensional inspection of highly reflective metallic objects is still a delicate task. We deal with a new automated three-dimensional inspection system based on polarization analysis. We first present an extension of the shape-from-polarization method for dielectric surfaces to metallic surfaces. Then, we describe what we believe to be a new way of solving the ambiguity concerning the normal orientation with an active lighting system. Finally, applications to shape-defect detection are discussed, and the efficiency of the system to discriminate defects on specular metallic objects made by stamping and polishing is presented. () 2006 Optical Society of America

OCIS code: 110.6880
\end{abstract}

\section{Introduction}

The three-dimensional (3D) machine-vision systems market is expanding rapidly, providing 3D-based machine vision for process control. Nevertheless, the inspection of specular metallic objects remains a delicate task, since it requires controlling the whole lighting of the scene. Extracting shape information of specular metallic surfaces from a single view is still a challenging problem, and there are currently two techniques. The first is studying the highlights of structured lightings, with multiple ring lights, ${ }^{1}$ with a hemisphere of 127 point sources like the SHINY system, ${ }^{2}$ or with a calibrated pattern composed of lines. ${ }^{3,4}$ The second technique is studying the motion of a specularly reflected pattern on the surface that is different from the real motion of the object. ${ }^{5}$ Here, we propose what we believe to be a new way of reconstructing the 3D information on specular metallic objects by extending the shape-from-polarization method to metallic surfaces. Koshikawa and Shirai ${ }^{6}$ first used polarization information to determine the shape of dielectric glossy objects. Shape information

The authors are with the Laboratoire Electronique, Informatiane et Image CNRS, UMR 5158, 12 rue de la Fonderie, 71200 Le

Creusot, France. O. Morel's e-mail address is o.morel@iutlecreusot. u-bourgogne.fr.

Received 7 September 2005; revised 23 November 2005; accepted 16 December 2005; posted 5 January 2006 (Doc. ID 64554).

0003-6935/06/170001-07\$15.00/0

(C) 2006 Optical Society of America was computed by analysis of the Stokes parameters of a circularly polarized light reflected by the object surface. Later, Wolff and Boult ${ }^{7}$ showed how the surface normals are constrained to the polarimetric parameters of an unpolarized light reflected by the surface. This shape-from-polarization method was developed by Miyazaki et al., 8,9 Rahmann, ${ }^{10}$ and Rahmann and Canterakis ${ }^{11}$ especially for transparent or reflective surfaces, but of a dielectric nature. By using the complex refractive index of the surface, we found new relations between the polarization images and the surface normals. ${ }^{12-14}$ The previous authors did not provide any robust solutions to solve the ambiguity concerning the determination of the normal azimuth angle. We propose here a new way of solving it by using an active lighting system. Once the surface normals are computed, the surface is reconstructed by integration.

The paper is organized as follows: the shape-frompolarization method extended to specular metallic surfaces is presented in Section 2. Section 3 describes the active lighting system for solving the ambiguity of the azimuth angle. The description of the whole acquisition system and the results are given in Section 4.

\section{Shape from Polarization for Metallic Surfaces}

The surface is assumed to be continuous and is described by a Cartesian expression: $z=f(x, y)$. Therefore each surface normal (Fig. 1) is given by the following nonnormalized expression: 


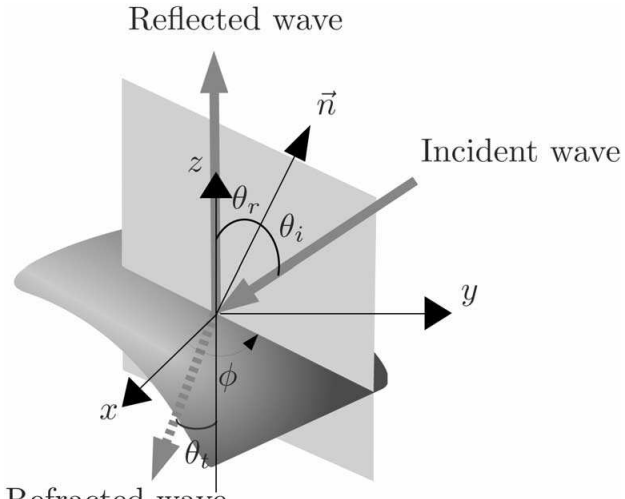

Refracted wave

Fig. 1. Light-wave reflection on a specular surface.

$$
\vec{n}=\left(\begin{array}{c}
-\frac{\partial f(x, y)}{\partial x} \\
-\frac{\partial f(x, y)}{\partial y} \\
1
\end{array}\right)=\left(\begin{array}{l}
p=\tan \theta_{r} \cos \phi \\
q=\tan \theta_{r} \sin \phi \\
1
\end{array}\right) .
$$

This expression establishes the relationship between the normal orientation (given by the zenith angle $\theta_{r}$ and the azimuth angle $\phi$ ) and the partial derivatives of the surface. This section presents the two main steps of the shape-from-polarization method. First, we demonstrate how to compute the surface normals (especially the metallic one), thanks to the polarization properties of the reflected light. Second, we describe an algorithm for reconstructing the 3D shape by integrating the normals.

\section{A. Polarization Imaging}

After being reflected, an unpolarized light wave becomes partially linearly polarized, depending on the surface normal and on the refractive index of the media it impinges on. A partially linearly polarized light wave has three parameters:

- light magnitude $I$,

- degree of polarization $\rho$,

- angle of polarization $\varphi$.

The degree of polarization varies from 0 for a completely unpolarized light to 1 for a perfectly linearly polarized light. To study the polarization state of such a wave, the use of a rotating polarizer in front of a camera is enough. ${ }^{15}$ The relationship between the magnitude $I_{p}$ of the transmission of a partially linearly polarized light wave through a rotating polarizer and the angle $\alpha$ of the polarizing filter is given by a sinusoid (Fig. 2):

$$
I_{p}(\alpha)=\frac{I_{\max }-I_{\min }}{2} \cos (2 \alpha-2 \varphi)+\frac{I_{\max }+I_{\min }}{2}
$$

where $I_{\min }$ and $I_{\max }$ represent, respectively, the minimum and the maximum magnitudes seen through

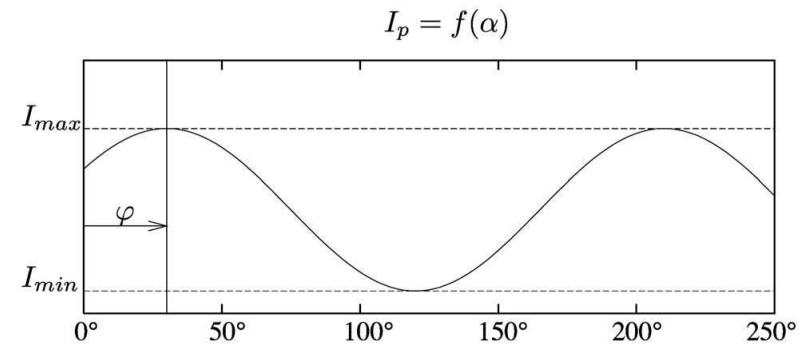

Fig. 2. Variation of the light intensity according to the angle of the polarizer $\alpha$.

the polarizer. Equation (2) can be written in the form

$$
I_{p}(\alpha)=\frac{I}{2}[\rho \cos (2 \alpha-2 \varphi)+1]
$$

with

$$
I=I_{\max }+I_{\min }, \quad \rho=\frac{I_{\max }-I_{\min }}{I_{\max }+I_{\min }} .
$$

The purpose of polarization imaging is to compute the three parameters, $I, \varphi$, and $\rho$, by interpolating formula (3). There are several methods for fitting the equation to get the parameters. Because there are three parameters, at least three images, taken with different orientations of the polarizer, are required. Since the degree of polarization is low for metallic surfaces, and to improve the accuracy of the measure of the parameters, we apply the linear least-squares method by taking 18 frames with a constant step of $10^{\circ}$.

1. Relationship Between the Angle of Polarization $\varphi$ and the Azimuth Angle $\phi$

Wolff and Boult have demonstrated how to determine constraints on surface normals by using the Fresnel reflectance model. The Fresnel coefficients of reflection $F_{\perp}$ and $F_{\|}$are the ratio between the amplitude of the reflected light and the incident light according, respectively, to the plane perpendicular and the plane parallel to the plane of incidence:

$$
\left.\begin{array}{l}
F_{\perp}=\left|\frac{\sin \left(\theta_{i}-\theta_{t}\right)}{\sin \left(\theta_{i}+\theta_{t}\right)}\right|^{2} \\
F_{\|}=\left|\frac{\tan \left(\theta_{i}-\theta_{t}\right)}{\tan \left(\theta_{i}+\theta_{t}\right)}\right|^{2}
\end{array}\right\},
$$

where $\theta_{i}$ is the angle of incidence and $\theta_{t}$ is the angle of refraction. Fresnel formulas (5) show that the orthogonal component $F_{\perp}$ is greater than the parallel one $F_{\|}$. Therefore it means that an unpolarized light wave becomes partially linearly polarized according to the normal of the plane of incidence. Thus the azimuth angle $\phi$ can be inferred from the angle of polarization $\varphi$ : 


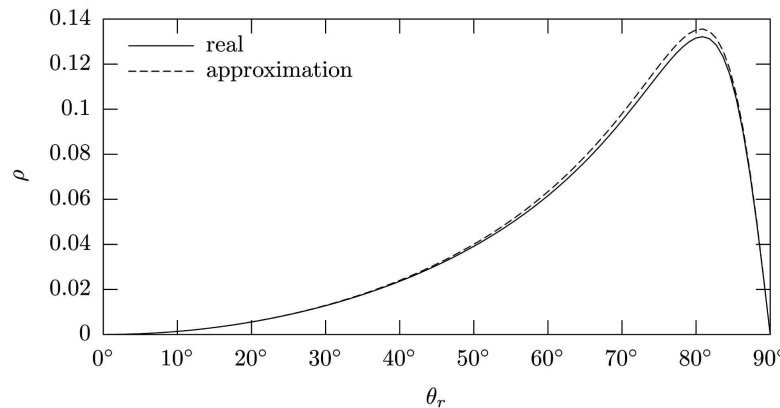

Fig. 3. Comparison between the approximated degree of polarization and the real degree of polarization for a metallic surface $(n=1.94+5.28 i)$.

$$
\phi=\varphi \pm \frac{\pi}{2}
$$

A new technique to disambiguate this relation is presented in the next subsection.

\section{Relationship Between the Degree of Polarization} $\rho$ and the Zenith Angle $\theta_{r}$

The disparity between the components $F_{\perp}$ and $F_{\|}$ brings also a new piece of information: the degree of polarization of the reflected light. Indeed, the degree of polarization can be expressed as follows:

$$
\rho=\frac{F_{\perp}-F_{\|}}{F_{\perp}+F_{\|}} .
$$

To find a new relationship between the degree of polarization and the zenith angle for metallic surfaces, we introduce the complex refractive index: $\hat{n}=n(1+i \kappa)$ (where $\kappa$ is called the attenuation index). By assuming the refractive index of the air to be 1, we can write the Snell-Descartes law:

$$
\sin \theta_{i}=\hat{n} \sin \theta_{t},
$$

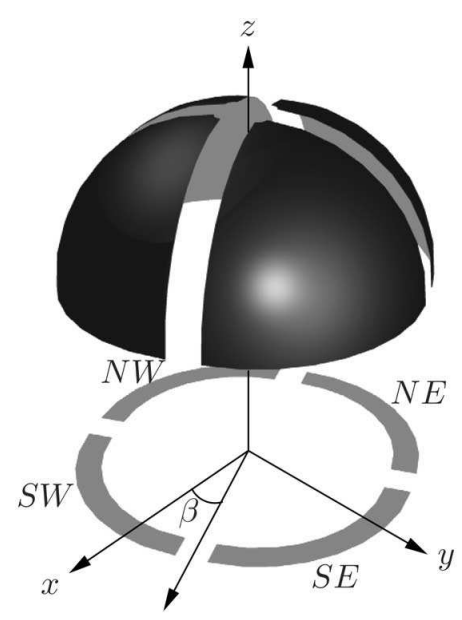

Fig. 4. (Color online) Expanded view of the active lighting system.

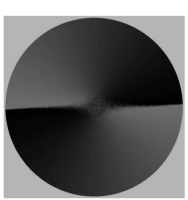

(a)

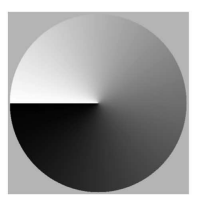

(b)

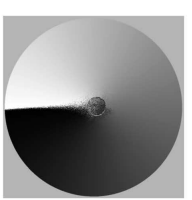

(c)
Fig. 5. Disambiguation of the azimuth angle: (a) without disambiguation ( $\phi$ is defined modulo $\pi$ ), (b) theoretical values ( $\phi$ is defined modulo $2 \pi$ ), (c) result of the disambiguation ( $\phi$ is defined modulo $2 \pi$ ).

where $\theta_{i}$ and $\theta_{t}$ are, respectively, the incident and the refractive angles. To simplify the relations, and as is usually the case in the visible region, the following approximation $^{16}$ is applied:

$$
|\hat{n}|^{2}=n^{2}\left(1+\kappa^{2}\right) \gg 1
$$

Thanks to this approximation, by substituting Eqs. (5) into Eq. (7) and by writing $\theta_{i}=\theta_{r}$ with SnellDescartes law (8), we can express the degree of polarization $\rho$ for metallic surfaces as follows:

$$
\rho\left(\theta_{r}\right)=\frac{2 n \tan \theta_{r} \sin \theta_{r}}{\tan ^{2} \theta_{r} \sin ^{2} \theta_{r}+|\hat{n}|^{2}} .
$$

Figure 3 shows that the difference between the true degree of polarization, and its approximation grows slightly only for great values of the angle $\theta_{r}$. The curve also shows that the ambiguity concerning the determination of the angle $\theta_{r}$, according to the degree of polarization $\rho$, does not have to be treated for surfaces with slopes lower than $80^{\circ}$, is the case with the studied objects. For most metallic surfaces, the peak appears generally at a higher angle than that for dielectric surfaces. Nevertheless, the disambiguation method developed by Miyazaki et al. ${ }^{9}$ for the angle $\theta_{r}$ can be applied. In this case, two degrees of polarization images have to be acquired with a light rotation of the object.

\section{B. Surface Reconstruction}

Once the angles $\theta_{r}$ and $\phi$ are determined, the normals are computed according to Eq. (1). To reconstruct the surface shape from the normals, we use the FrankotChellappa $^{17}$ algorithm. Denoting by $\tilde{f}, \tilde{p}$ and $\tilde{q}$ the Fourier transforms of, respectively, the surface height and the $x, y$ gradients, we have

$$
\forall(u, v) \neq(0,0), \quad \tilde{f}(u, v)=\frac{-j u \tilde{p}-j v \tilde{q}}{u^{2}+v^{2}}
$$

We reconstruct the surface shape by taking the inverse transform of the former equation. As we can see, this algorithm is available only for surfaces with a null mean slant. Since the derivative and the Fourier transform are linear operators, the surface can be split according to: 


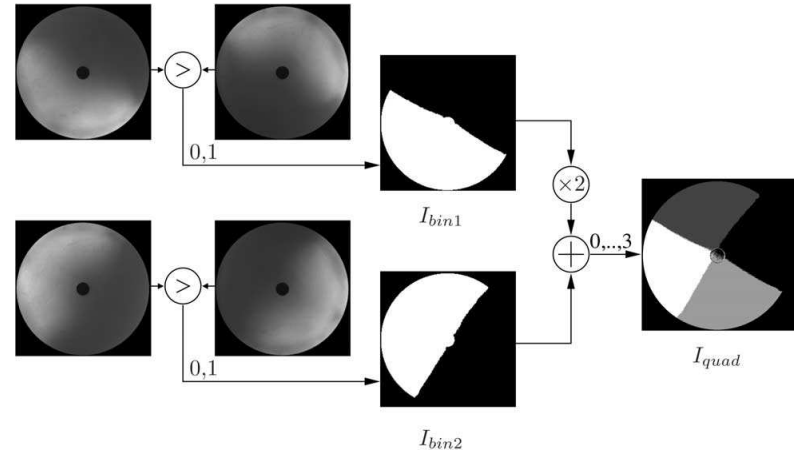

Fig. 6. Acquisition principle of the segmented image $I_{\text {quad }}$.

$$
f(x, y)=f_{0}(x, y)+g(x, y)
$$

where $f_{0}$ is the surface reconstructed from Eqs. (11) and $g$ is the surface mean plane; $g$ can be deduced by

$$
g(x, y)=x \tilde{p}(0,0)+y \tilde{q}(0,0)+\mathrm{cst}
$$

where cst is a constant (integration constant).

This algorithm based on a Fourier transform is less time consuming than the standard algorithm based on relaxation. ${ }^{18}$ The shape for $1024 \times 1024$ sized images are built in less than $1 \mathrm{~s}$.

\section{Active Lighting System}

The previous section showed the ambiguity about the determination of the azimuth angle $\phi$ from the angle of polarization $\varphi$. Miyazaki et al. ${ }^{9}$ numerically solve this ambiguity by propagating the information at the occluding boundary to the rest of the object. It implies obtaining the whole object image and assuming that the object is geometrically closed and that all local parts of the surface are not concave toward the camera direction. To solve this ambiguity we propose a new active lighting system.

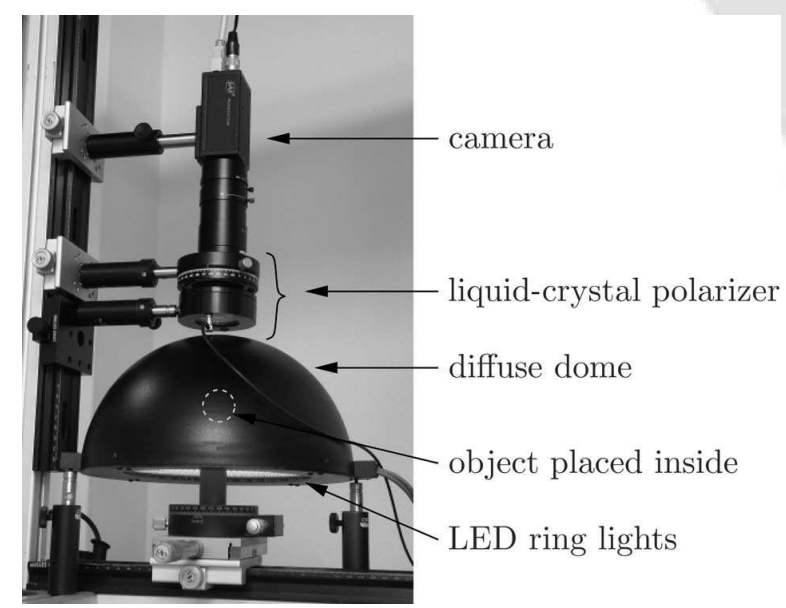

Fig. 7. Experimental setup.

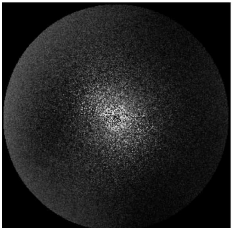

(a)

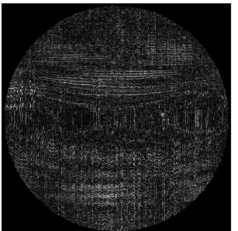

(b)

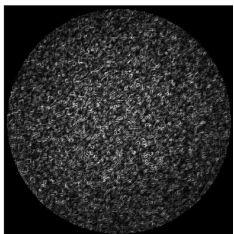

(c)

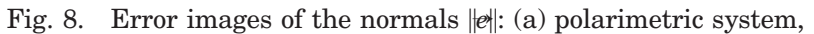
(b) Replica scanner, (c) Minolta VI-910 scanner.

\section{A. Description}

Our lighting system is a diffuse dome light (an expanded view is presented on Fig. 4) composed of a ring with numerous LEDs and a dome whose surface is diffuse. It provides a uniform and unpolarized light onto the object to be digitized. The ring of LEDs is split into four parts that can be independently electrically controlled. The offset orientation of the dome $\beta$ belongs to the interval $[0, \pi / 2]$.

\section{B. Principle}

Relation (6) between the angle of polarization $\varphi$ and the azimuth angle $\phi$ can be expressed as

$$
\phi=\varphi-\pi / 2+\left\{\begin{array}{l}
0 \\
\pi
\end{array}\right.
$$

To demonstrate ambiguity solving, a hemisphere was used because it enables us to clearly represent all the possible surface orientations. Since the azimuth angle $\phi$ is not linked to the refractive index, the azimuth angle image of the hemisphere can be computed nearly all over the surface. Figures 5(a) and 5(b) show the azimuth angle images of the hemisphere, from our acquisition system (without disambiguation) and from the theoretical model, respectively. As one can see, $\pi$ has to be added to the azimuth angle of the points whose normals are oriented to the north. The splitting of the LED ring into two parts would have been sufficient to discriminate the north and south orientations, but it requires a perfect orientation of the dome light: $\beta=0$.

To disambiguate the azimuth angle $\phi$, we first compute a segmented image $I_{\text {quad }}$ that discriminates the surface points whose normals are oriented according to the NW, NE, SW, or SE directions. Figure 6 describes the acquisition principle of the segmented image $I_{\text {quad }}$ for the hemispheric object. The binary image $I_{\text {bin } 1}$ that discriminates the east and west orientations is obtained by a comparison of the intensity images acquired with the east and west lighting. In the same way, the binary image $I_{\text {bin2 } 2}$ is obtained by a comparison of the intensity images acquired with the south and north lighting. The segmented image $I_{\text {quad }}$ that discriminates the four orientations is computed according to the relation $I_{\text {quad }}=2 I_{\text {bin } 1}+I_{\text {bin2 } 2}$. Second, $\pi$ has to be added to the azimuth angle $\phi$ whose normals are oriented according to the north direction. Thus, from the azimuth image $\phi[$ Fig. 5(a)] and from 
Table 1. Mean Error on Normals Acquisition

\begin{tabular}{cccc}
\hline $\begin{array}{c}\text { Hemisphere } \\
\text { Diameter }(\mathrm{mm})\end{array}$ & $\begin{array}{c}\text { Polarimetric } \\
\text { System }\end{array}$ & $\begin{array}{c}\text { Replica } \\
\text { Scanner }\end{array}$ & $\begin{array}{c}\text { Minolta } \\
\text { Scanner }\end{array}$ \\
\hline 41.275 & 0.0695 & 0.0882 & 0.1034 \\
44.450 & 0.0644 & 0.0614 & 0.0790 \\
\hline
\end{tabular}

the segmented image $I_{\text {quad }}, \pi$ has to be added to the points for which

- $I_{\text {quad }}=0$ and $\phi \leq 0$,

- $I_{\text {quad }}=1$,

- $I_{\text {quad }}=3$ and $\phi \geq 0$.

Finally, the algorithm of the disambiguation process can be described according to these steps:

1. $\phi=\varphi-\pi / 2$,

2. Computing $I_{\text {quad }}$,

3. If $\left[\left(I_{\text {quad }}=0\right) \wedge(\phi \leq 0)\right] \vee\left[I_{\text {quad }}=1\right] \vee\left[\left(I_{\text {quad }}\right.\right.$ =3) $\wedge(\phi \geq 0)$, then $\phi=\phi+\pi$,

where $\wedge$ and $\vee$ represent, respectively, the logical operators AND and OR. The result of the disambiguation is presented Fig. 5(c).

\section{Experiments}

\section{A. Experimental Setup}

The acquisition system is made up of a CCD camera, a liquid-crystal polarization rotator, and an active diffuse dome light (Fig. 7). The active diffuse dome light is used both to provide an unpolarized light on the whole surface and to solve the ambiguity concerning the azimuth angle. Since the target object is highly specular, it is placed in the center of the dome to be illuminated on most of its surface. After reflection, the light that becomes partially linearly polarized is studied with the liquid-crystal polarization rotator and the camera. The liquid-crystal polarization rotator acts as a rotating polarizer, which can be electrically controlled. This device uses nematic liquid-crystal cells associated with a linear polarizer and a quarter-wave plate, which provides tunable polarization by changing the supplied voltage. Since the degree of polarization is lower for metallic sur-

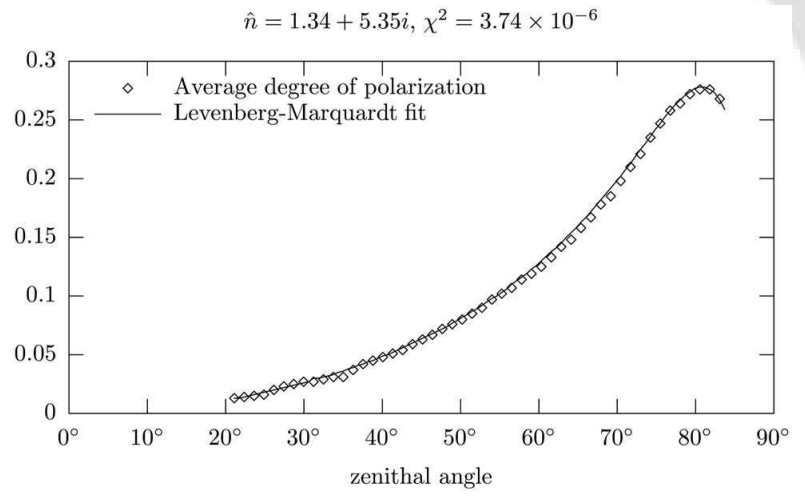

Fig. 9. Calibration curve.

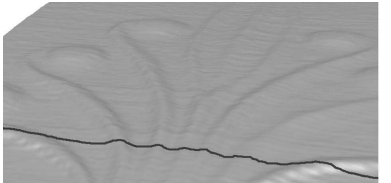

(a)

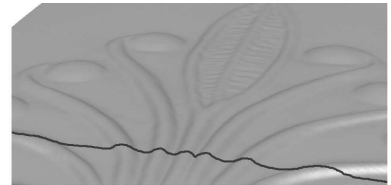

(b)
Fig. 10. 3D reconstruction of a specular metallic object: (a) surface from the 3D scanner, (b) surface from our method.

faces, we use a sensitive camera with a 10-bit depth. A limitation of the system is due to the hole for the camera, leading to a $\theta_{\min }$ value that is about $2^{\circ}$. Therefore the object has to be correctly oriented to respect the constraints about the angle $\theta_{r}$. Nevertheless, approximating the angle $\theta_{r}$ as inferior to $2^{\circ}$ to $\theta_{r}=0^{\circ}$ (optical axis orientation) does not lead to a significant error in the $3 \mathrm{D}$ surface reconstruction.

\section{B. Quantitative Comparisons}

The 3D reconstruction of surfaces is linked to both the acquisition system accuracy and the object shape. Thus, instead of comparing the shape reconstructed from different devices, we compared the normals. To compare the accuracy of the normals acquisition, calibrated hemispheres were used. The norm of the error defined by the following relation was used:

$$
\|\vec{e}\|=\left\|\vec{n}_{\mathrm{th}}-\vec{n}_{m}\right\|
$$

where the subscripts th and $m$ indicate, respectively, the theoretical and measured data.

Figure 8 shows error images taken from three different systems: our polarimetric system, the Replica 500 scanner, and the Minolta VI-910 scanner. A perfectly specular metallic hemisphere, with a refractive index equal to $\hat{n}=1.94+5.28 i$, was used with our system whereas a mat hemisphere was used with the two scanners based on triangulation. The norm of the error is computed with angle $\theta_{r}$ in the interval $\left[0,80^{\circ}\right]$, meaning that not all the hemisphere surface normals are computed for the three systems in Fig. 8. The error from the polarimetric system is quite random and homogeneous on the surface except on the center, where the normals are oriented near the optical axis. It is due to both the hole in the dome and to the degree of polarization that becomes very small in this region. Indeed, in this case the reflection co-

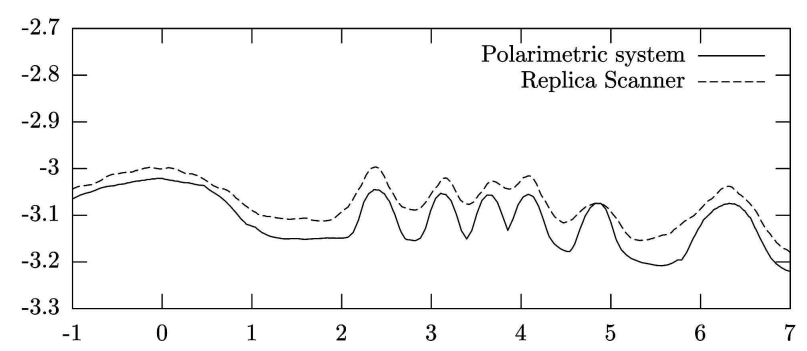

Fig. 11. Comparison of the cross sections (scales given in millimeters). 


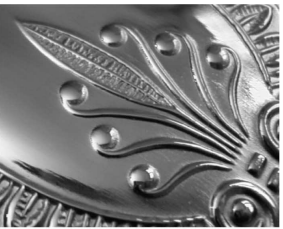

(a)

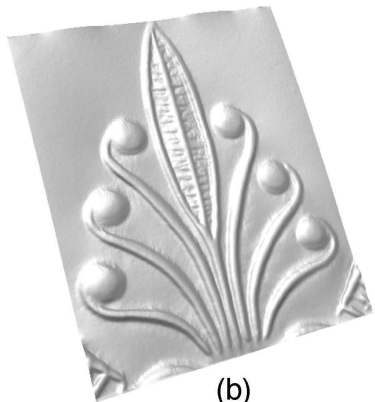

(b)
Fig. 12. (a) Object reference photograph and (b) its 3D reconstructed surface.

efficients are nearly the same, increasing slightly an uncertainty concerning the angle $\phi$. Table 1 presents the normal mean errors with $\theta_{r} \in\left[0,80^{\circ}\right]$ for two different sized hemispheres and shows the good accuracy of our system for computing the surface normals.

\section{Qualitative Comparisons}

To qualitatively compare a $3 \mathrm{D}$ surface obtained with our system with a 3D surface of reference, we scanned the object with the Replica 500 scanner. This scanner, based on laser ranging, takes regular points with steps of $50 \mu \mathrm{m}$ in the $X$ and $Y$ axes, and the precision in the $Z$ axis is about $20 \mu \mathrm{m}$. The material of the object being studied is stainless steel. In computing the angle $\theta$ from the degree of polarization, two issues are possible. The first one, presented in Section 2, is to determine directly the angle $\theta$ by knowing the refractive index of the object material. The second one is to estimate a pseudorefractive index that enables the best fit of the relation between the angle $\theta$ and the degree of polarization measured on a known shape of the same material. To determine this index, a known shape of the same material is placed in the center of the dome and the degree of polarization is measured. Then a calibration curve, that represents the measured average degree of polarization according to the zenith angle can be created (Fig. 9). Finally, the Levenberg-Marquardt nonlinear algorithm is used to estimate a pseudorefractive index that best fits the data. This pseudore-

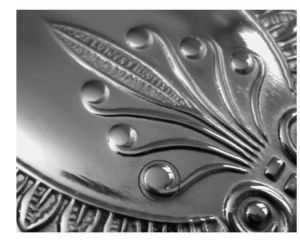

(a)

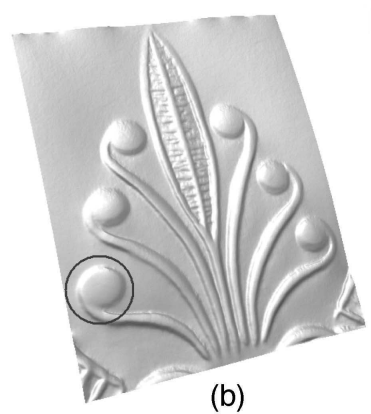

(b)
Fig. 13. (a) Photograph of an object with a shape defect and (b) its $3 \mathrm{D}$ reconstructed surface $(\mathrm{b})$.

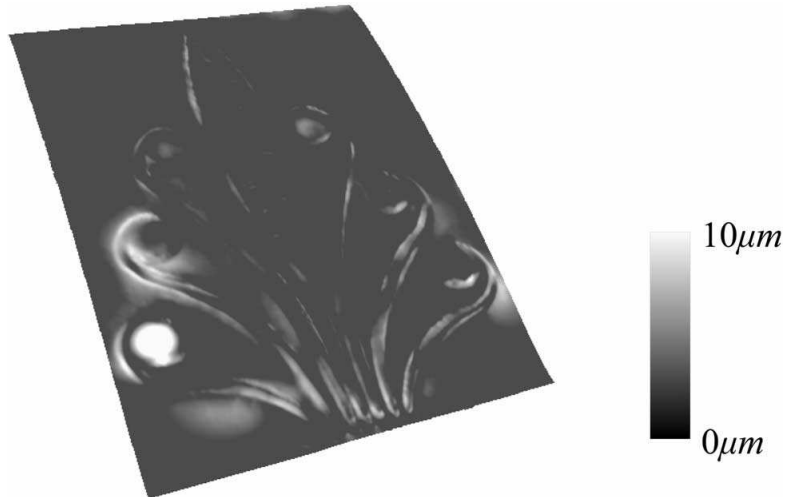

Fig. 14. Mean deviation between the two reconstructed surfaces.

fractive index, which has no physical meaning, enables us to compute the zenith angle image of the new object by using relation (10).

Since the Replica scanner is sensitive to the reflectivity of the surface, a thin opaque coating has to be applied onto the object. Consequently the details of the object appear clearly more marked with our method than those with the scanner [Figs. 10(b) and 10(a), respectively]. After registering the two surfaces, the mean deviation between the surfaces is about $30 \mu \mathrm{m}$, meaning that the shape is qualitatively well reconstructed. Moreover, the cross section, presented in Fig. 11, highlights the good accuracy of the surface computed with our method. The resolutions along the $X$ and $Y$ axes depend on only the lenses used and on the spatial resolution of the sensor: For instance, we are able to get $X$ and $Y$ resolutions that are three times finer compared with those obtained with the Minolta scanner.

\section{Application to Three-Dimensional Inspection}

The 3D inspection of highly reflective metallic surfaces is still a delicate task in industrial vision. The aim of our system is to reveal the shape defects on decorations from metallic objects made by stamping and polishing [Fig. 12(a)]. Previous work carried out by Seulin et al. ${ }^{19}$ was efficient in discriminating geometric aspect defects thanks to dynamic lighting: when a fringe pattern was projected, defects on a smooth surface were revealed near the transition between dark fringes and bright fringes. Nevertheless, this system is not adapted for inspecting shape defects on the object's decorations. With our polarimetric system, the $3 \mathrm{D}$ surface of a reference object is computed [Fig. 12(b)]. The 3D surface of the objects to be inspected is computed and compared with the reference one. For instance, Fig. 13 shows the photograph and the reconstructed surface of an object with a shape defect on the left-hand-bottom bump. After the two shells are registered, the mean deviation between the surfaces is computed (Fig. 14). In this way, our system efficiently discriminates shape defects. The ambiguity problem that appears on the bumps (because normals orientations change from the south 
to the north) was previously manually solved.12-14 Results shown here present automatic 3D surface reconstruction, thanks to the active lighting system.

\section{Conclusions}

In this paper, a new way of reconstructing the 3D surface of specular metallic objects has been presented. The shape-from-polarization method has been extended to specular metallic surfaces. By using a complex refractive index, and thanks to an approximation commonly used in optics, we found new relations for computing the normals. Moreover, the active lighting system we developed enables us to solve the ambiguity concerning the normal orientation. This new way of disambiguating the azimuth angle is more robust than the numerical methods. Finally, the involved process is quite simple, requiring an active diffuse dome light, a CCD camera, and a polarization rotator. The different parts are all electrically controlled, and the 3D surfaces are automatically computed in less than $5 \mathrm{~s}$ with the presented configuration: 18 polarization images $(1024 \times 1024)$. This acquisition time can be drastically reduced by use of faster liquid-crystal polarization rotators. The efficiency of the polarimetric system for computing the $3 \mathrm{D}$ shape is also presented. An application for inspecting shape defects on metallic specular objects is described. The comparison between a reference surface and a three-dimensional surface to be inspected reveals shape defects. The registration between the two surfaces, required before the comparison, is not automatic yet. Therefore future works will consist of including an automatic registration in the system. ${ }^{20,21}$

\section{References}

1. I. Yun, E. Jung, and S. Lee, "On the fast shape recovery technique using multiple ring lights," Pattern Recogn. 30, 883-893 (1997).

2. S. Nayar, A. Sanderson, L. Weiss, and D. Simon, "Specular surface inspection using structured highlight and Gaussian images," IEEE Trans. Rob. Autom. 6, 208-218 (1990).

3. S. Savarese and P. Perona, "Local analysis for 3D reconstruction of specular surfaces," in IEEE Computer Vision and Pattern Recognition (IEEE, 2001), Vol. 2, pp. 738-745.

4. S. Savarese and P. Perona, "Local analysis for 3D reconstruction of specular surfaces: Part II," in Proceeding of the European Conference on Computer Vision (2002), pp. 759-774.

5. J. Zheng and A. Murata, "Acquiring a complete 3D model from specular motion under the illumination of circular-shaped light sources," IEEE Trans. Pattern Anal. Mach. Intell. 22, 913-920 (2000).

6. K. Koshikawa and Y. Shirai, "A model-based recognition of glossy objects using their polarimetrical properties," Adv. Rob. 2, 137-147 (1987).

7. L. B. Wolff and T. E. Boult, "Constraining object features using a polarization reflectance model," IEEE Trans. Pattern Anal. Mach. Intell. 13, 635-657 (1991).

8. D. Miyazaki, M. Kagesawa, and K. Ikeuchi, "Determining shapes of transparent objects from two polarization images," in Proceedings of the IAPR Workshop on Machine Vision Applications, (IAPR, 2002), pp. 26-31.

9. D. Miyazaki, M. Kagesawa, and K. Ikeuchi, "Transparent surface modeling from a pair of polarization images," IEEE Trans. Pattern Anal. Mach. Intell. 26, 73-82 (2004).

10. S. Rahmann, "Inferring 3D scene structure from a single polarization image," Proc. SPIE 3826, 22-33 (1999).

11. S. Rahmann and N. Canterakis, "Reconstruction of specular surfaces using polarization imaging," in IEEE Computer Vision and Pattern Recognition (IEEE, 2001), Vol. 1, pp. 149155.

12. O. Morel, C. Stolz, and P. Gorria, "Application of polarimetric imaging to 3D inspection of highly reflective metallic surface," Proc. SPIE 5606, 82-89 (2004).

13. O. Morel, C. Stolz, F. Meriaudeau, and P. Gorria, "Polarization imaging applied to 3D inspection of specular metallic surfaces," in Proc. SPIE 5679, 178-186 (2005).

14. O. Morel, C. Stolz, F. Meriaudeau, and P. Gorria, "Threedimensional inspection of highly-reflective metallic objects by polarization imaging," Electron. Imag. Newslett. 15(2), 4 (2005).

15. L. B. Wolff, "Polarization vision: A new sensory approach to image understanding," Image Vision Comput. 15, 81-93 (1997).

16. M. Born and E. Wolf, Principles Of Optics, 7th ed. (Cambridge U. Press, 1999).

17. R. Frankot, and R. Chellappa, "A method for enforcing integrability in shape from shading algorithms," IEEE Trans. Pattern Anal. Mach. Intell. 10, 439-451 (1988).

18. K. Ikeuchi, "Determining surface orientations of specular surfaces by using the photometric stereo method," IEEE Trans. Pattern Anal. Mach. Intelli. 3, 661-669 (1981).

19. R. Seulin, F. Merienne, and P. Gorria, "Simulation of specular surface imaging based on computer graphics: application on a vision inspection system," EURASIP J. Appl. Signal Process. 2002, 649-658 (2002).

20. P. Besl and N. McKay, "A method for registration of 3-d shapes," IEEE Trans. Pattern Anal. Mach. Intelli. 14, 239-256 (1992).

21. C. Matabosch, J. Salvi, D. Fofi, and F. Meriaudeau, "Range image registration for industrial inspection," in Proc. SPIE 5679, 216-227 (2005). 